\title{
Slotting y picking: una revisión de metodologías y tendencias
}

\author{
Slotting and Picking: A review of methodologies and trends
}

\author{
Juan Camilo Duque Jaramillo ${ }^{1} \quad$ Manuela Cuellar Molina $^{1} \quad$ Juan Miguel Cogollo Flórez ${ }^{2 *}$ \\ Recibido 15 de septiembre de 2018, aceptado 25 de marzo de 2019 \\ Received: September 15, 2018 Accepted: March 25, 2019
}

\begin{abstract}
RESUMEN
La gestión de cadenas de suministro implica la formulación de modelos y metodologías de mejora y optimización de operaciones y procesos internos, con el fin de aumentar la eficiencia y la capacidad de respuesta y a su vez reducir costos. En la gestión de cadenas de suministro, las actividades llevadas a cabo en almacenes y centros de distribución son fundamentales para garantizar un nivel de servicio óptimo y para obtener ahorros significativos en los costos logísticos totales. El slotting y el picking son dos actividades fundamentales en la operación y administración eficiente de las instalaciones de almacenamiento. Por ello, en este artículo se hace una revisión bibliográfica exhaustiva sobre los modelos y metodologías usados en la optimización de dichas actividades entre los años 2000 y 2018, identificando aplicaciones indirectas en otro tipo de industrias y oportunidades y tendencias de investigación, considerando los factores que influyen en los flujos de materiales y componentes estocásticos en la planeación de inventarios: acomodo dinámico, análisis de rutas, metodologías de ubicación de clústeres y división de almacén por tipo de productos.
\end{abstract}

Palabras clave: Slotting, picking, almacenamiento, mejora, optimización.

\begin{abstract}
Supply Chain Management is based on formulating models and methodologies to improve and optimize operations and internal processes, in order to increase efficiency and response capacity and simultaneously reduce costs. In Supply Chain Management, activities carried out in warehouses and distribution centers are critical to ensure an optimum service level and to obtain significant savings in total logistics costs. Slotting and picking are two fundamental activities in the efficient warehouses operation and management. For this reason, we made in this article an exhaustive bibliographic review on models and methodologies used for optimizing slotting and picking, between 2000 and 2018, identifying indirect applications in other types of industries and research opportunities and trends, considering the factors that influence the flow of materials and stochastic components in inventory planning: dynamic accommodation, route analysis, cluster location methodologies and warehouse division according to the type of products.
\end{abstract}

Keywords: Slotting, picking, warehousing, improvement, optimization.

1 Universidad Nacional de Colombia. Facultad de Minas. Departamento de Ingeniería de la Organización. Medellín, Colombia. E-mail: jcduquej@unal.edu.co; macuellarmo@unal.edu.co

2 Instituto Tecnológico Metropolitano - ITM. Departamento de Calidad y Producción. Medellín, Colombia.

E-mail: juancogollo@itm.edu.co

* Autor de correspondencia: juancogollo@itm.edu.co 


\section{INTRODUCCIÓN}

Una cadena de suministro es un sistema organizacional en el cual sus miembros actúan como un todo para garantizar un flujo constante de bienes, fondos e información financiera y operacional entre los diferentes eslabones [1]. Así, la gestión de cadenas de suministro involucra el diseño y gestión de todas las actividades de una cadena de suministro para garantizar la satisfacción del cliente y maximizar la rentabilidad de la cadena [2]. Para ello, se parte de la definición de estrategias, métodos, actividades y metodologías para el funcionamiento de la cadena de suministro; luego, el objetivo es lograr el funcionamiento eficiente y eficaz de los diferentes eslabones, en especial, reducir tiempos de espera y optimizar el uso de los recursos existentes para disminuir los costos de operación individuales y de la cadena de suministro [3-6].

En el funcionamiento de los almacenes, las actividades de recogida, acomodo y recuperación de pedidos podrían representar entre el $55 \%$ y el $65 \%$ de los costos totales [7], por ello, una mejora en dicho proceso, podría conllevar a un ahorro significativo en los costos de operación y, por ende, en el costo logístico total [8]. Estas mejoras, también afectan las medidas de desempeño de cada una de las áreas involucradas en la satisfacción de los clientes, tales como almacenes, líneas de producción, administración, entre otros, logrando así aumentar no solo la competitividad propia, sino llevar a una consolidación, estructuración y desarrollo de su cadena de suministro [9-11].

Uno de los factores fundamentales que restringe la optimización del funcionamiento y mejora de almacenes es el diseño que este posea y su flexibilidad en cuanto a condiciones y niveles de uso. Inicialmente, en una planeación realizada por parte del área logística para definir las condiciones que deben tener los almacenes, de acuerdo con las necesidades y proyecciones de la compañía, se establecen las restricciones referentes a su uso, capacidad, espacio y otras características fundamentales. Una vez analizadas las posibles alternativas a elegir, a nivel estratégico y táctico y se tome y ejecute la decisión, se convertirá en restricciones rígidas para su administración.

El diseño de almacenes, usualmente, se basa en el proceso productivo, las necesidades de materias primas y sus respectivos espacios para ser almacenadas, incluyendo en ocasiones los espacios necesarios para almacenar producto terminado [12]. Este diseño, además, considera factores esenciales como la proyección de crecimiento industrial en ciertos periodos de tiempo, la demanda de productos estacionales, entre otros, incluyendo la manera en que fluyen los SKUs (Stock Keeping Units) de acuerdo a la cantidad y frecuencia de rotación, con el objetivo de obtener una configuración más flexible y que se adapte a las necesidades reales del sistema de almacenamiento [12-13].

A pesar de lo mencionado anteriormente, se puede encontrar una desventaja con relación al control del flujo de SKUs en el sistema logístico, dado que al tener variaciones de las cantidades sujetas a la oferta, demanda, ciclo de vida y otras características de los productos y el mercado, haría necesario un replanteamiento del funcionamiento del sistema de inventarios, teniendo presente que cuando se diseña el almacén, en la mayoría de los casos se mantiene constante dichas condiciones en el mediano o largo plazo. Luego de establecer las políticas de inventarios, de almacén y definir los parámetros relacionados con las dimensiones y configuración, se debe proceder a desarrollar una estrategia para realizar las actividades internas del almacén, entre las que se encuentran principalmente el slotting y el picking.

Por ello, en este artículo se hace una revisión bibliográfica exhaustiva sobre los modelos y metodologías usados en la optimización de slotting y picking entre los años 2000 y 2018 , identificando aplicaciones indirectas en otro tipo de industrias y oportunidades y tendencias de investigación. La estructura del artículo se resume de la siguiente forma: en la siguiente sección se presenta el marco teórico o referente conceptual. Después, se describe la metodología usada para la realización de la revisión sistemática de literatura. Luego, se presentan los resultados obtenidos, resaltando aspectos clave, clasificación y análisis de las metodologías de optimización y mejora de slotting y picking encontradas en la literatura, al igual que se mencionan algunas aplicaciones en sectores y operaciones diferentes al almacenamiento. Finalmente, se mencionan tendencias y oportunidades de investigación en estas temáticas, con base en el desarrollo teórico y los hallazgos encontrados. 


\section{MARCO TEÓRICO}

El slotting es considerado como una actividad del área de logística consistente en la distribución de artículos (materia prima, componentes y otros materiales del proceso o producto) entre los almacenes, en caso de ser varios, y al interior de estos. Esta distribución tiene como objetivo fundamental acomodar los SKUs en los espacios dedicados o slots, de manera que se minimicen los tiempos de desplazamiento y los espacios de uso. Para esto, se han desarrollado diversas técnicas, métodos y algoritmos que buscan alcanzar el máximo desempeño del almacén en relación con los objetivos planteados por la empresa [12-13].

El picking se enfoca en la preparación de los pedidos de las órdenes en los almacenes para ser entregados. Según Zhang [14], el picking consiste, en primer lugar, en recibir la orden en el almacén, luego el encargado ("picker"), bien sea un sistema automatizado o un operario recolector, se dirige a la isla o bloque de estantes donde se encuentre el SKU solicitado, realiza la recolección y analiza si la orden está completa; en caso de ser así, se dirige al punto de entrega; de lo contrario, analiza su capacidad de transporte de los distintos SKU y se dirige al siguiente SKU hasta completar la orden que posteriormente es llevada al punto de acopio, bien sea para consolidar con otra fracción del pedido o ser enviada a su destino.

Los dos problemas centrales en que se enfocan las metodologías de optimización y mejoras en el funcionamiento interno de los almacenes tienen los siguientes enfoques [15]: (i) Definir la ubicación en que se debe situar cada SKU, esto es, el slotting, y, (ii) Definir la ruta de recolección de SKUs de una orden, esto es, el picking. Además de estos, existen otros enfoques, correspondientes al diseño del almacén, que se centran en la distribución de los "huecos" (slots) o estanterías, optimización de uso de espacios [16], unificación de diferentes SKUs mejorando el uso volumétrico, entre otros [13].

Una característica presente en la mayoría de los modelos presentados para dar solución a los problemas de slotting y de picking es el uso de condiciones determinísticas. Al obtener una solución o estrategia muy rígida, se pueden generar sobrecostos que están asociados directa o indirectamente al dimensionamiento del almacén. También se debe tener presente, que las empresas establecen sus medidas de desempeño en relación con los objetivos planteados. Una de las medidas más usadas es la distancia de viaje promedio por picking [14], teniendo presente que al reducir estas distancias se podrían reducir tiempos y costos de procesamiento y aumentar la eficiencia del sistema.

\section{METODOLOGÍA}

Con el fin de identificar las contribuciones más importantes que se han realizado al estudio de slotting y picking y sus tendencias de investigación, se realizó una revisión exhaustiva de literatura, la cual permitió identificar, interpretar y sintetizar la evidencia documentada en el periodo entre los años 2000 y 2018, siguiendo las siguientes etapas [17]: (1) Formulación de preguntas de investigación, (2) Búsqueda en bases de datos, (3) Definición de criterios de inclusión y exclusión, y (4) Análisis de resultados.

En esta sección se describen las tres primeras etapas y en la próxima sección se muestra la etapa cuatro de análisis de resultados. Así, en la primera etapa se formularon las preguntas de investigación que guiaron el desarrollo del estudio:

- P1: ¿Cuáles modelos/metodologías de análisis de slotting y picking se usan en la industria actualmente?

- P2: ¿Qué tipo de aplicaciones de los modelos/ metodologías de slotting y/o picking se han hecho en operaciones diferentes al almacenamiento de artículos?

- P3: ¿Cuáles son las tendencias u oportunidades de investigación en los métodos de optimización y mejora de slotting y picking?

Luego, en la segunda etapa, se llevó a cabo el proceso de búsqueda en las bases de datos Science Direct, IEEE y Emerald, en el periodo de tiempo mencionado anteriormente y usando las siguientes palabras clave: "slotting and picking", "slotting and warehouse", "picking and warehouse" y "slotting and picking and warehouse".

En la tercera etapa se llevó a cabo la selección de la evidencia documental pertinente a las preguntas de investigación planteadas, considerando tres 
criterios de inclusión: (i) Presenta aporte teórico sobre slotting y/o picking, (ii) Presenta propuesta o aplicación de modelos de slotting y/o picking, (iii) Presenta caso de aplicación de modelos conjuntos de slotting y picking. En forma análoga, se excluyeron los artículos que tenían alguna de las siguientes características (criterios de exclusión): (i) Presentan desarrollo teórico sobre slotting y/o picking en áreas de conocimiento diferentes a logística o administración de cadenas de suministro y (ii) Presenta desarrollo de modelos de slotting y/o picking no relacionados con la administración o diseño de almacenes.

\section{RESULTADOS}

\section{Resultados de la búsqueda}

Aplicando los criterios de búsqueda y considerando las bases de datos previamente mencionadas, se recuperaron un total de 5540 artículos. Luego, con la aplicación de los criterios de inclusión y exclusión, se seleccionaron 36 artículos definitivos. En la Figura 1 se presentan la cantidad de artículos de las revistas con mayor número de publicaciones con respecto a los temas de interés dentro del periodo de tiempo definido. Luego, en la Figura 2 se presenta la cantidad de publicaciones por año con respecto a las diferentes ecuaciones de búsqueda en las mismas revistas, en la que se puede evidenciar una tendencia de crecimiento sobre investigaciones y desarrollo de la temática. También, en la Figura 3, se presenta la cantidad de artículos de acuerdo a las diferentes ecuaciones de búsqueda conjuntas ("Slotting and picking; "Slotting and Warehouse"; "Picking and warehouse"; "Slotting and picking and warehouse"), con el fin de poder observar la tendencia de las series inferiores de la Figura 2.

Con el fin de dar respuesta a la primera pregunta de investigación sobre modelos y metodologías de análisis de slotting y picking que se usan en la industria actualmente y lograr un análisis más exhaustivo de los artículos recuperados y sus aportes a la temática en estudio, los 36 artículos seleccionados fueron clasificados usando un enfoque taxonómico de dos niveles [18] (Tabla 1). El primer nivel comprende las siguientes categorías: (1) Tipo de técnica, (2) Tipo de contribución, (3) Tipo de slotting/picking, (4) Aplicación del modelo, (5) Características de los datos y (6) Sector de aplicación.

Dentro de cada categoría, los artículos son caracterizados en una o varias de las subcategorías presentadas. La primera categoría, tipo de técnica,

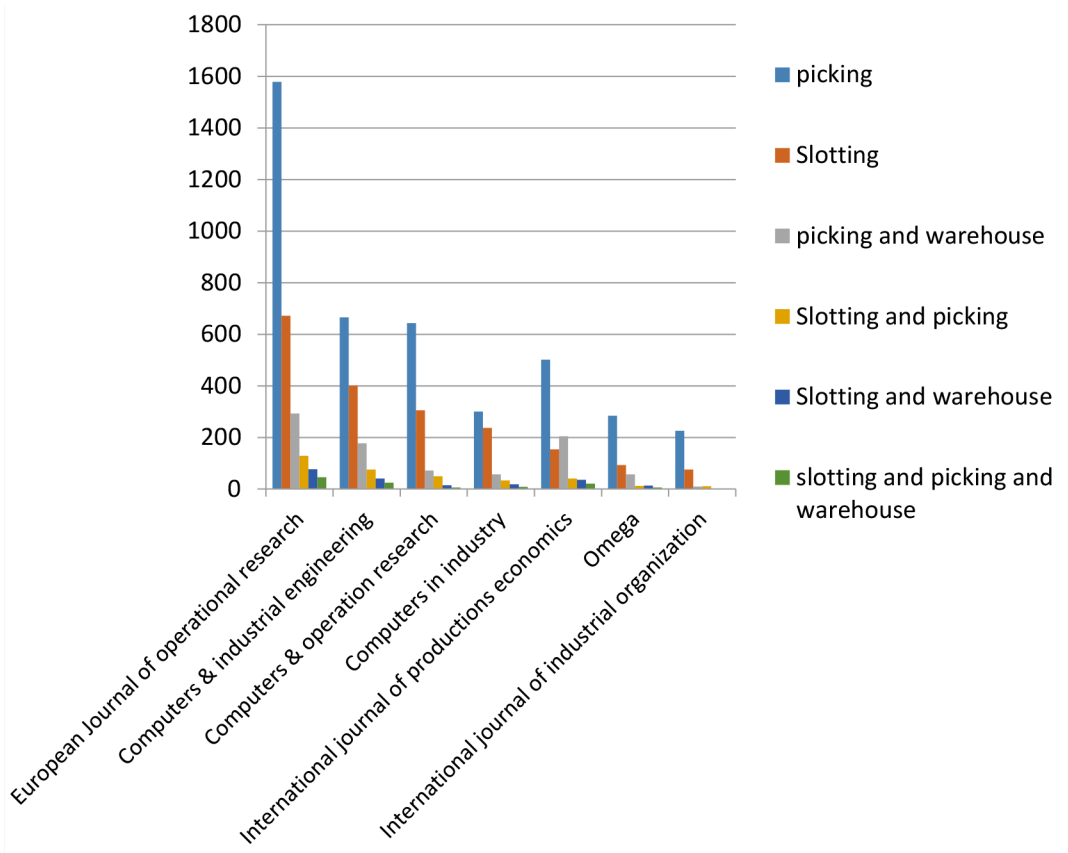

Figura 1. Cantidad de artículos por revista en periodo 2000-2018. 


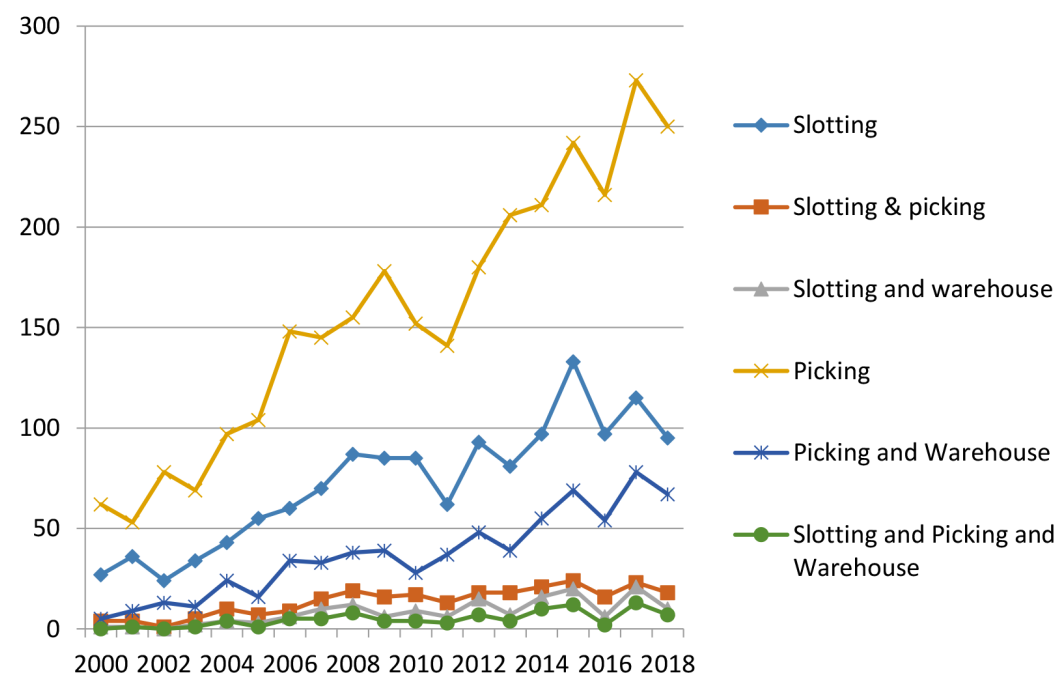

Figura 2. Publicaciones por año de acuerdo con las ecuaciones de búsqueda.
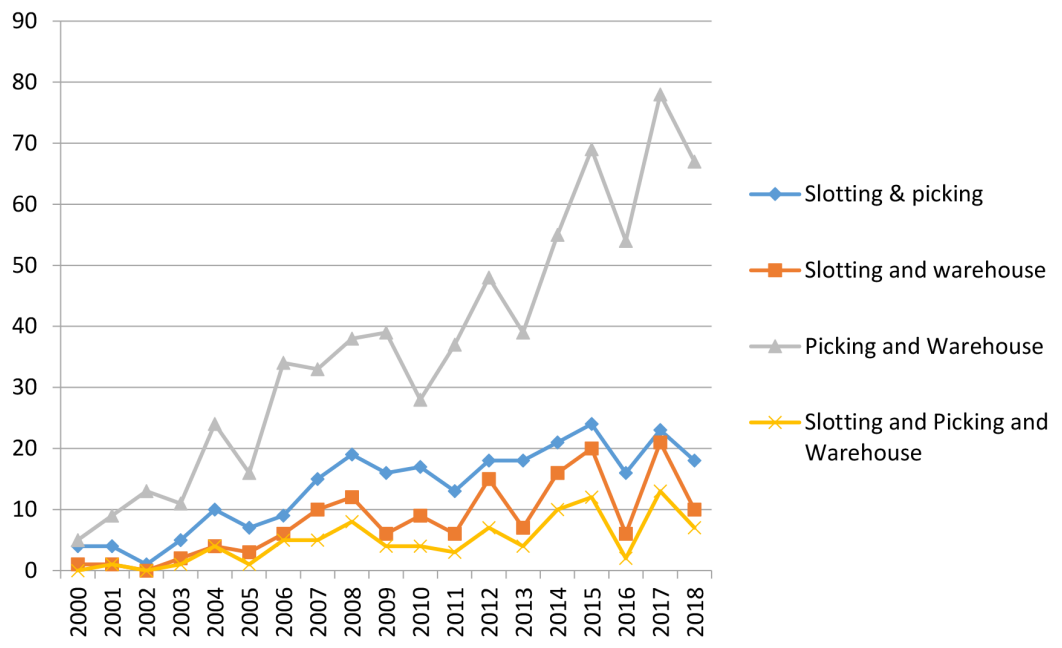

Figura 3. Publicaciones por año de acuerdo con las ecuaciones de búsqueda conjuntas.

comprende la clasificación principal de cada artículo de acuerdo con el área de estudio en la que se enfoca (slotting, picking o warehouse). La segunda categoría, tipo de contribución, hace referencia al aporte teórico o analítico. La tercera categoría, tipo de slotting/picking, comprende las subcategorías referidas al tipo de almacenamiento, características de los artículos e interrelación con otras operaciones de almacenamiento. La cuarta categoría, aplicación del modelo, se refiere a si el modelo propuesto fue aplicado o no. La quinta categoría, características de los datos, se refiere al uso de datos reales o sintéticos o el no uso de datos. La última categoría, sector de aplicación, comprende los diferentes sectores económicos o industriales donde se aplican los modelos propuestos.

El cumplimiento de los atributos de cada subcategoría se marca con una "X" en las celdas respectivas. En la Tabla 1 se observa que las subcategorías marcadas solo una vez constituyen el $10 \%$, no hay subcategorías marcadas solo dos veces y las subcategorías marcadas tres veces corresponden al 5\%. Por tanto, el 85\% de las subcategorías están marcadas cuatro o más veces, 
Tabla 1. Clasificación taxonómica de los artículos seleccionados.

\begin{tabular}{|c|c|c|c|c|c|c|c|c|c|c|c|c|c|c|c|c|c|c|c|c|}
\hline \multirow[b]{2}{*}{ 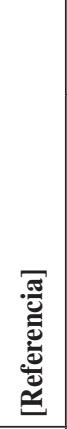 } & \multicolumn{3}{|c|}{$\begin{array}{l}\text { 1. Tipo de } \\
\text { técnica }\end{array}$} & \multicolumn{2}{|c|}{$\begin{array}{l}\text { 2. Tipo de } \\
\text { contribución }\end{array}$} & \multicolumn{5}{|c|}{$\begin{array}{l}\text { 3. Tipo de Slotting / } \\
\text { Picking }\end{array}$} & \multicolumn{2}{|c|}{$\begin{array}{l}\text { 4. Aplicación } \\
\text { del modelo }\end{array}$} & \multicolumn{3}{|c|}{$\begin{array}{l}\text { 5. Características } \\
\text { de los datos }\end{array}$} & \multicolumn{5}{|c|}{$\begin{array}{l}\text { 6. Sector } \\
\text { de aplicación }\end{array}$} \\
\hline & 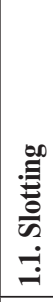 & 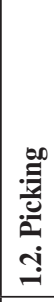 & 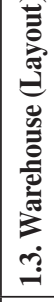 & 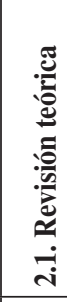 & 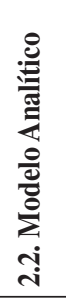 & 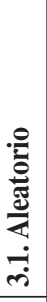 & 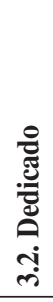 & 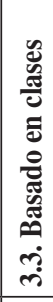 & 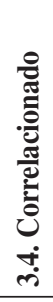 & 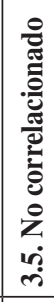 & 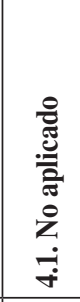 & 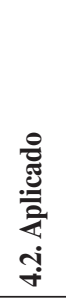 & 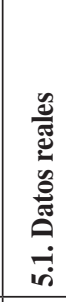 & 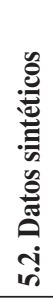 & 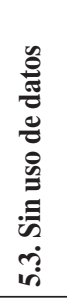 & 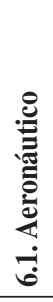 & 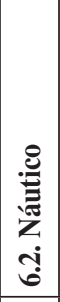 & 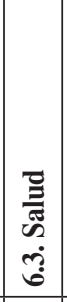 & 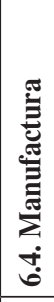 & 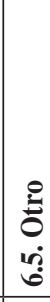 \\
\hline [3] & $X$ & & $X$ & & $X$ & $\mathrm{X}$ & & & & & & $\mathrm{X}$ & & $X$ & & & & & & $X$ \\
\hline [4] & $X$ & & & & $X$ & $\mathrm{X}$ & $X$ & & & & & $\mathrm{X}$ & $X$ & & & $X$ & & & & \\
\hline [5] & $X$ & & & & $\mathrm{X}$ & & & $X$ & & & & $\mathrm{X}$ & $X$ & & & & & & $X$ & \\
\hline [6] & $X$ & $X$ & & & $X$ & & & & $X$ & & & $\mathrm{X}$ & & $X$ & & & & & $X$ & \\
\hline [7] & & $X$ & & & $X$ & & & & & $X$ & & $X$ & $X$ & & & & & & $X$ & \\
\hline [9] & & $X$ & & & $\mathrm{X}$ & & & & & $\mathrm{X}$ & & $\mathrm{X}$ & & $\mathrm{X}$ & & & & & $X$ & \\
\hline [10] & $X$ & $X$ & $X$ & & $X$ & & & $X$ & $\mathrm{X}$ & & & $X$ & & $\mathrm{X}$ & & & & & $X$ & \\
\hline [11] & $\mathrm{X}$ & & $\mathrm{X}$ & & $\mathrm{X}$ & $X$ & & & & & $\mathrm{X}$ & & & & $\mathrm{X}$ & & & & $X$ & \\
\hline [12] & $X$ & $X$ & & $X$ & & $X$ & $X$ & $X$ & & $X$ & $X$ & & & & $X$ & & & & & $X$ \\
\hline [13] & & $X$ & $X$ & $\mathrm{X}$ & & & & & & $X$ & $\mathrm{X}$ & & & & $X$ & & & & & $X$ \\
\hline [15] & $X$ & & & & $X$ & $X$ & $\mathrm{X}$ & & & & $\mathrm{X}$ & & & & $\mathrm{X}$ & & & & $X$ & \\
\hline [16] & $X$ & & X & & $\mathrm{X}$ & & $\mathrm{X}$ & & & & & $\mathrm{X}$ & & $X$ & & & & & $X$ & \\
\hline [14] & $X$ & $\mathrm{X}$ & & & $X$ & & & & $X$ & $\mathrm{X}$ & & $X$ & & $\mathrm{X}$ & & & & & $X$ & \\
\hline [19] & $X$ & $X$ & & & $\mathrm{X}$ & & & & $X$ & & & $\mathrm{X}$ & $X$ & & & & & & & $\mathrm{X}$ \\
\hline [20] & $X$ & $\mathrm{X}$ & $\mathrm{X}$ & $X$ & $\mathrm{X}$ & $X$ & $X$ & & & $\mathrm{X}$ & & $\mathrm{X}$ & & $\mathrm{X}$ & & & & & & $\mathrm{X}$ \\
\hline [21] & $\mathrm{X}$ & & & $\mathrm{X}$ & & & $X$ & & & & $\mathrm{X}$ & & & & $\mathrm{X}$ & & & & & $\mathrm{X}$ \\
\hline [22] & $X$ & & & & $\mathrm{X}$ & & & $X$ & & & & $\mathrm{X}$ & $X$ & & & & & & & $\mathrm{X}$ \\
\hline [23] & $X$ & & & $X$ & & & & $X$ & & & $X$ & & & & $\mathrm{X}$ & & & & & $\mathrm{X}$ \\
\hline [24] & $X$ & & & $X$ & & & & $\mathrm{X}$ & & & $\mathrm{X}$ & & & & $\mathrm{X}$ & & & & & $\mathrm{X}$ \\
\hline [25] & $\mathrm{X}$ & $\mathrm{X}$ & & & $\mathrm{X}$ & & & & $X$ & & & $\mathrm{X}$ & $X$ & & & & & & $X$ & \\
\hline [26] & $X$ & $X$ & & & $\mathrm{X}$ & & & & $X$ & & & $\mathrm{X}$ & $X$ & & & & & & $X$ & \\
\hline [27] & $X$ & $\mathrm{X}$ & & $\mathrm{X}$ & $\mathrm{X}$ & $X$ & $X$ & $\mathrm{X}$ & $\mathrm{X}$ & & & $\mathrm{X}$ & $\mathrm{X}$ & & & & & & $\mathrm{X}$ & \\
\hline [28] & $\mathrm{X}$ & $\mathrm{X}$ & & & $\mathrm{X}$ & & & $\mathrm{X}$ & $X$ & & $\mathrm{X}$ & & & & $\mathrm{X}$ & & & & $X$ & \\
\hline [29] & $X$ & $\mathrm{X}$ & $X$ & & $\mathrm{X}$ & & & & $X$ & & & $\mathrm{X}$ & $X$ & & & & & & $X$ & \\
\hline [30] & $X$ & & $X$ & & $\mathrm{X}$ & & $\mathrm{X}$ & & & & & $\mathrm{X}$ & $X$ & & & & & & & $\mathrm{X}$ \\
\hline [31] & $X$ & & & & $\mathrm{X}$ & & & $\mathrm{X}$ & & & $\mathrm{X}$ & & & & $\mathrm{X}$ & $\mathrm{X}$ & & & & \\
\hline [32] & $\mathrm{X}$ & & & & $\mathrm{X}$ & & $\mathrm{X}$ & & & & & $\mathrm{X}$ & $X$ & & & & $\mathrm{X}$ & & & \\
\hline [33] & $X$ & & & $X$ & & & $X$ & & & & $X$ & & & & $\mathrm{X}$ & $X$ & & & & \\
\hline [34] & $X$ & & & $\mathrm{X}$ & & & $X$ & & & & $\mathrm{X}$ & & & & $\mathrm{X}$ & & & $X$ & & \\
\hline [35] & & $X$ & $X$ & & $\mathrm{X}$ & & & & & $X$ & & $\mathrm{X}$ & $X$ & & & & & & $X$ & \\
\hline [36] & $X$ & & $X$ & & $\mathrm{X}$ & $X$ & $X$ & & & & & $\mathrm{X}$ & & $\mathrm{X}$ & & & & & $X$ & \\
\hline [37] & & $X$ & $X$ & $X$ & $\mathrm{X}$ & & & & & $\mathrm{X}$ & & $\mathrm{X}$ & & $X$ & & & & & $X$ & \\
\hline [38] & $X$ & $X$ & $X$ & & $X$ & & & & $X$ & & & $\mathrm{X}$ & & $X$ & & & & & $X$ & \\
\hline [39] & $X$ & $X$ & $X$ & $X$ & & & & & $X$ & & & $\mathrm{X}$ & $X$ & & & & & & $X$ & \\
\hline$[40]$ & & $X$ & $\mathrm{X}$ & & $\mathrm{X}$ & & & & & $\mathrm{X}$ & & $\mathrm{X}$ & $\mathrm{X}$ & & & & & & $X$ & \\
\hline [41] & & $\mathrm{X}$ & $X$ & & $\mathrm{X}$ & & & & & $\mathrm{X}$ & & $\mathrm{X}$ & $X$ & & & & & & $X$ & \\
\hline
\end{tabular}


lo que permite afirmar que la taxonomía desarrollada es robusta para clasificar concisamente los artículos seleccionados sobre modelos y metodologías de análisis de slotting y picking.

En cuanto a los resultados de la clasificación taxonómica, la mayoría de artículos (86\%) son contribuciones sobre slotting y el $36 \%$ corresponde a metodologías de análisis conjunto de slotting y picking. En cuanto al tipo de contribución, el 70\% de los artículos corresponde a modelos analíticos estrictos y el $8 \%$ plantea tanto modelos analíticos como una importante contribución teórica. Los porcentajes de las contribuciones en lo referente a los tipos de slotting/picking son aproximadamente similares, lo cual indica que no hay preponderancia por un tipo específico, sino que se estudian en una proporción equivalente. La mayoría de los modelos son aplicados $(69,4 \%)$, el $41,7 \%$ usando datos reales y el $27,7 \%$ usando datos sintéticos. Finalmente, en lo referente al sector de aplicación, se evidencia la preponderancia del sector manufacturero $(58 \%$ de los modelos), aunque hay aplicaciones en sectores emergentes como el aeronáutico, náutico y salud (14\% en conjunto).

\section{Clasificación de las metodologías}

Con base en lo planteado anteriormente, se realizó una clasificación de las metodologías de slotting y picking, identificando los autores de las principales contribuciones, la cual se presenta en la Figura 4. Este enfoque de perfilado se basa en la clasificación de slotting propuesta por Bindi, Manzini, Pareschi y Regattieri [19], donde se tienen cuatro categorías: almacenamiento aleatorio, dedicado, basado en clases y correlacionado. El picking presenta una categoría de clasificación de "correlacionado", que corresponde a la planeación conjunta de picking y slotting, dado que el slotting correlacionado se aplica con el fin de reducir no solo distancias y tiempos de acomodamiento [14], sino también la recolección de pedidos. El picking no correlacionado corresponde a las metodologías de picking que no tienen como base o complemento directo las actividades de slotting.

En los siguientes apartados se detallan las principales contribuciones identificadas en la revisión de literatura sobre metodologías de slotting y picking, basado en el perfil de la Figura 4.

\section{Tipos de slotting}

Las actividades de slotting se clasifican de acuerdo al tipo de almacenamiento y características de los artículos almacenados, así [14, 19]:

- Almacenamiento aleatorio: A cada SKU que entra al almacén se le asigna una ubicación aleatoria, teniendo en cuenta la disponibilidad de espacio,

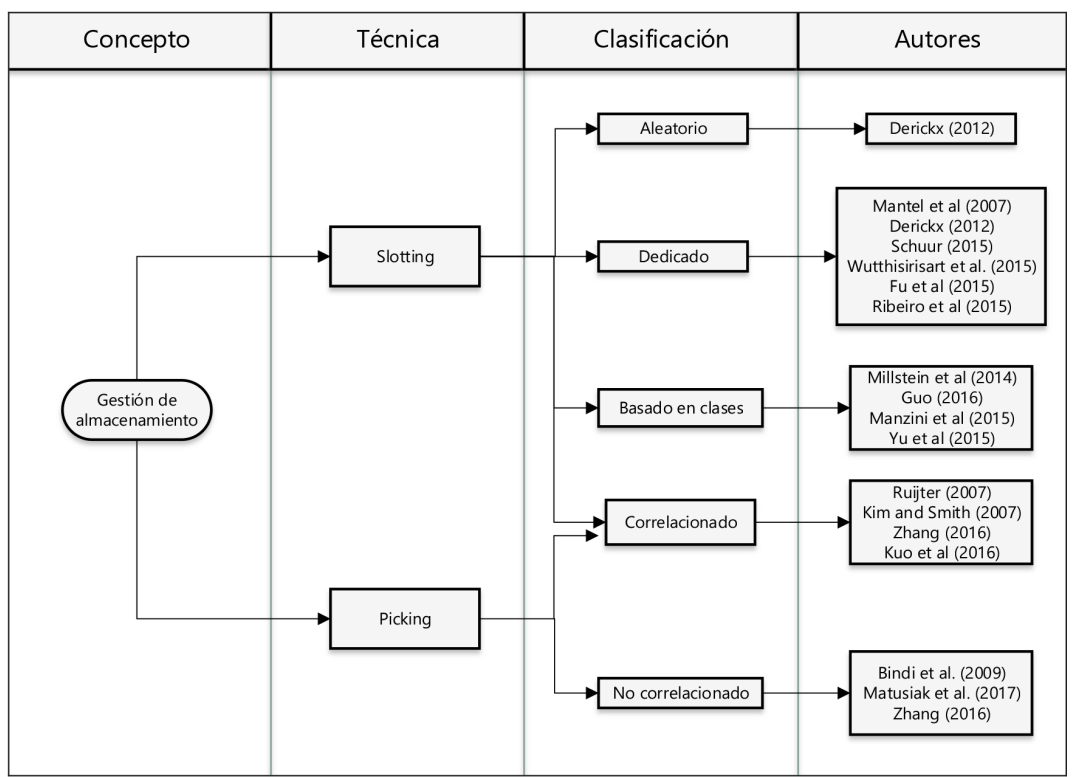

Figura 4. Clasificación de metodologías de Slotting y Picking. 
o por experiencia [20]; esto puede ocasionar consecuencias negativas en el desempeño dado que no se tiene mayor control de ubicación de inventarios al interior del almacén y podría conllevar a costos mayores, sobre todo en el caso de productos perecederos.

- Almacenamiento dedicado: Se enfoca en la asignación específica de los SKUs a los slots, dándoles así, una ubicación clara de dónde irá cada SKU [20]. Dentro de este tipo de almacenamiento, se encuentra la estrategia de slotting Cube per Order Index (COI; Índice Cúbico de Pedido, en español), propuesto por Heskett en 1963, metodología que aún se encuentra vigente y es presentada en libros de logística y administración de cadena de suministro [42]. También, Schuur [21] desarrolla la idea de que realizar las actividades de slotting basándose en el COI no siempre es la mejor opción, dado que no considera muchos factores que terminarían afectando el desempeño del almacén como las herramientas de transporte, distancias, entre otros; por lo cual, se debe analizar qué factores son críticos en el desarrollo de dichas actividades, cuáles se mantiene constantes y cómo podrían influenciar cada uno de ellos en las medidas de desempeño.

- Almacenamiento basado en clases: Consiste en clasificar los SKUs en diferentes clases, considerando criterios como rotación, popularidad, entre otros [22, 23]. Usualmente, se usa la clasificación $A B C$, que se fundamenta en la teoría de Pareto. Millstein, Yang y Li [5] desarrollaron una herramienta informática con el fin de optimizar el número de agrupaciones de SKU y a su vez mejorar los niveles de desempeño, añadiéndole herramientas de experimentos computacionales y análisis de costos. Por otra parte, Yu, De Koster y Guo [23] desarrollan un análisis sobre la clasificación por clases en la que se muestra que no siempre es conveniente usar mayor cantidad de clases, dado que podría conllevar a una asignación no eficiente de los SKU y afectar el desempeño. Finalmente, Guo [24] presenta un análisis de las políticas de almacenamiento, donde se destaca la programación de la estrategia $\mathrm{ABC}$ en los sistemas de control.

- Almacenamiento correlacionado: Su criterio de decisión es el agrupamiento de los SKUs en "clusters", es decir, un agrupamiento entre piezas y cantidades de acuerdo con las necesidades correlacionadas de dos o más SKUs, analizando bajo las órdenes de preparación de pedidos, cómo se piden estos en conjunto y otros criterios [25]. Es en este tipo de metodologías, donde el picking se toma como criterio para la asignación de los espacios, puesto que no solo es de interés el primer flujo de los productos en el almacén (desde su llegada hasta su ubicación en el espacio de almacenamiento), sino que además considera su recolección y preparación de los pedidos.

\section{Métodos de slotting}

La aplicación de los métodos de slotting para la localización de los SKUs en el almacén se pueden analizar desde varios puntos de vista, donde una medición inicial justificada en la literatura o un comportamiento observado en la práctica sirve como soporte a la decisión de ubicación de SKUs de manera interna. Los métodos de slotting con aplicaciones más comunes en la industria, se presentan a continuación:

- ABC: Consiste en la clasificación y almacenamiento de los productos en tres grupos (A, B o C), de acuerdo a las curvas de demanda, que se construyen basados en la participación o rotación de cada uno de los productos [24]; para esto, usualmente se realiza una gráfica de proporción de demanda acumulada en unidades, contra la proporción de ítems almacenados [23]. Los productos clasificados tipo A, se almacenan cercanos a los puntos de entrada o de salida, debido a su alta rotación, mientras que los productos tipo $\mathrm{C}$, que representan el menor flujo interno, tienden a almacenarse más lejos, o inclusive, en otro almacén [42].

- COI: También conocido como "Cube per Order Index". Fue desarrollado por Hesket en 1963, el cual se enfoca en la rotación de volumen de los SKUs [42], es decir, combina los aspectos de popularidad de los SKUs con su volumen. Se centra básicamente en la frecuencia en que cada tipo de SKUs es requerido por unidad de espacio ocupada [21]. También se puede aplicar slotting por índice de popularidad o rotación y por índice volumétrico, pero son poco considerados debido a que el COI considera ambas características en conjunto.

- Ubicación por correlación entre SKUs: esta estrategia tiene como factor de decisión de 
ubicación las diferentes correlaciones entre SKUs o productos, es decir, que se debe realizar un análisis de las órdenes entrantes y encontrar cómo se piden los productos en conjunto con el fin de ubicarlos más cerca entre ellos y así disminuir la distancia máxima de viaje de recolección [14]. Esta estrategia se debe aplicar en dos pasos: el primero corresponde a un uso de matrices de correlaciones y luego, se debe tener en cuenta cuáles son los productos que más rotación representan, logrando así una ubicación estratégica de los SKUs con mayor rotación, pero, a su vez, cerca de los productos con los que suele pedirse. Esta también podría ser llamada ubicación por clústeres, pero usando zona completa para un producto en algunos casos [25], donde se asignan islas o espacios para la materia prima de cada producto final; a pesar de esto, sería eficiente en situaciones donde la cantidad de productos ofrecidos sea reducida, pues en gran cantidad de productos finales, llega a ser menos eficiente.

- Otras ubicaciones empíricas: son aplicadas, en la mayoría de los casos, cuando no se encuentran tecnificados los procesos. Este tipo de ubicación parte de la experiencia de administradores o empleados, quienes además de tener presentes restricciones de productos complementarios, compatibles y no compatibles, entre otras limitaciones, encuentran una distribución de los SKUs en el almacén sin respaldo técnico.

A pesar de que se ha demostrado en la práctica que dichos métodos de slotting se desempeñan bien, la mayoría de ellos no considera restricciones como la no compatibilidad entre productos, lo cual puede llevar a que la estrategia diseñada para localización de SKUs no sea válida y, por tanto, se deba replantear.

\section{Tipos de picking}

Las actividades de picking se clasifican de acuerdo con el grado de interrelación con otras operaciones de almacenamiento, así:

- Picking correlacionado: para el picking correlacionado se retoma la definición presentada por Kim y Smith [26]: "aquel en el que los SKUs que son tomados juntos en un mismo pedido, también deberían ser ubicados cerca". Con esto, se presenta una de las primeras formas de ubicación de SKUs y el picking se planea de forma complementaria al slotting. Los pasos de esta metodología se resumen en dos fases: agrupar en clústeres y luego asignar dichos clústeres a los espacios disponibles [27].

- Picking no correlacionado: hace referencia a la planeación de dicha actividad de manera independiente del slotting, siendo así el complemento de las metodologías de slotting por almacenamiento aleatorio, dedicado, o basado en clases. Mantel, Schuur y Heragu [28] desarrollan la estrategia llamada Order Oriented Slotting (OOS), donde se plantea una política de ruteo y preparación de pedidos. Por otra parte, Matusiak et al. [7] presentan una metodología y aplicación en la que se consideran las habilidades de cada "picker" en el desempeño del almacén.

\section{Métodos de picking}

Una vez establecidas las ubicaciones de los SKUs, se implementan algunos métodos de picking para optimizar las rutas de recolección. En este caso, son más usuales los métodos heurísticos, debido a las amplias posibilidades de combinaciones que se podrían tener entre productos $\mathrm{y}$, en consecuencia, la gran variedad de posibles órdenes en el sistema $[13,25,29]$.

En el estado del arte presentado por Zhang [14], se hace mención a algunos métodos heurísticos desarrollados para solucionar dicho problema:

- Modelo matemático y dos heurísticos directos con dos algoritmos genéticos híbridos con diferentes mecanismos de desarrollo considerando el conteo de peso de los SKUs.

- Heurísticos de asignación de almacenamiento modificando el heurístico basado en clases y la asociación de un heurístico basado en semilla, para optimizar las distancias recorridas en recolección de SKUs.

- Solución heurística en dos fases consistente en un algoritmo de retraso mínimo y una generación de diseño en las condiciones de cada columna o bahía que contiene un solo SKU [30].

Para el problema de slotting, se tienen presente las consideraciones básicas o restricciones generadas por las decisiones tomadas con respecto al diseño de almacenes, donde se define la capacidad, cantidad de slots y disponibilidad de los productos. Tanto para el problema de slotting como de picking, 
se pueden mejorar con las posibles soluciones presentadas en la literatura, pero que serían fácil de aplicar en ocasiones donde las cantidades de SKUs y el almacén sea considerablemente bajo, mientras que cuando se manejan grandes cantidades y volúmenes de referencias de productos, se hace necesario recurrir a uso de algoritmos genéticos o métodos heurísticos que permitan definir tanto la ubicación como las rutas de recolección, tal que garanticen un desempeño considerablemente bueno, aunque no sea el óptimo.

\section{Alternativas cuando se está al límite de capacidad Se deben tener en cuenta las posibles variaciones futuras cuando se realiza la proyección y planeación de diferentes almacenes, considerando crecimientos en demanda, crecimientos esperados e inesperados en participación e industria y otras condiciones que limitarán las decisiones y funcionamientos futuros. Debido a que los pronósticos presentan incertidumbre, es probable la presencia de posibles estados futuros que pueden generar gran impacto, tanto beneficios como complicaciones para las empresas.}

Uno de estos escenarios corresponde a la subutilización del espacio de almacenamiento, que se podría presentar por un estancamiento de la industria, o un decrecimiento por condiciones de mercado. En este caso el inconveniente principal corresponde a los sobrecostos generados por un sobredimensionamiento.

El segundo posible escenario, es que haya un crecimiento mayor al esperado, de modo que el almacén no tenga toda la disponibilidad para trabajar con la capacidad requerida, generando problemas internos como búsquedas de espacios de acumulación, saturación del almacén, entre otros. Para este escenario, los costos agregados al sistema corresponderían a aumentar las capacidades de almacenamiento, lo cual se puede hacer bajo dos alternativas principales: aumentar capacidad interna construyendo un nuevo almacén, o rentar uno [30].

En este caso, se pueden presentar varias metodologías de funcionamiento y se deben tomar decisiones sobre qué productos almacenar en cuál almacén, teniendo en cuenta factores como rotación y necesidades de producto, costos de transporte, ubicación del nuevo almacén, además si será propio o rentado [30], llevando a un planteamiento de administración de dos almacenes, pero que a medida que la cantidad de almacenes aumenta, su administración se vuelve más compleja ya que no solo deben tener control sobre ellos, sino cómo se trasladan los SKUs entre almacenes y con la planta de producción o zonas de ventas. Esta es una debilidad o falencia que tienen la mayoría de los modelos presentados, donde se asume un solo almacén.

\section{Otros casos de aplicación de slotting y picking}

Esta sección corresponde a la respuesta de la segunda pregunta de investigación sobre aplicaciones de modelos y metodologías de slotting y picking en operaciones diferentes al almacenamiento. $\mathrm{La}$ aplicación de slotting y picking no se restringe netamente a operaciones de almacenamiento en empresas del sector manufacturero o industrial. También se pueden realizar aplicaciones bajo sus principios en industrias como náutica y aeronáutica [4, 31, 32], u otras empresas de servicios. A continuación, se presentan algunas formas de aplicación.

\section{Industria aeronáutica}

Con respecto a la industria aeronáutica, existen dos actividades fundamentales para la aplicación de slotting. La primera, consiste en la coordinación de pistas y de espacios de parqueo de los aviones en un aeropuerto; para esto, se tiene como aspecto fundamental una coordinación interna eficiente para el desarrollo de actividades y que debe considerar la holgura de temporadas con climas no aptos para vuelo. La aplicación de slotting consiste en la asignación de los espacios de parqueo de los aviones teniendo presente dos factores principales: espacio necesario para su ubicación y fechas programadas de salida [4].

La segunda aplicación corresponde al almacenamiento de equipaje en aviones comerciales. A medida que se incrementan los tiempos de viaje y las escalas de abordaje, se requiere una adecuada distribución y ubicación de maletas dentro de las bodegas, para optimizar los tiempos de intercambios de equipaje entre aviones [33], teniendo presente que se deben descargar maletas para ser trasladadas, unas a otro avión y otras a las cintas de recolección de equipaje.

En el caso de importaciones y exportaciones, los procedimientos son similares, aunque usualmente en estos últimos servicios, se tiende a cargar y descargar total o parcialmente con mayores ventanas de tiempo. 


\section{Industria náutica}

La aplicación de slotting y picking juega un papel importante en el funcionamiento de los puertos marítimos y se puede analizar desde el mismo punto de vista utilizado en la industria aeronáutica. La diferencia radica en las mercancías transportadas y los volúmenes: podrían considerarse los contenedores como los SKUs a recoger o almacenar [32]. El funcionamiento de los puertos marítimos es similar a la industria aeronáutica, aunque tiene mayor similitud con casos de aplicaciones relacionadas con la industria manufacturera.

\section{Hospitales}

La aplicación indirecta de slotting en un hospital corresponde a la distribución de zonas internas como las áreas de hospitalización, unidades de cuidados intensivos, entre otras. En este caso, los flujos no son artículos, sino personas que deben ser atendidas y se debe disponer de espacios en salas de espera, habitaciones de hospitalización y cubículos para su respectiva atención y procedimientos. Así, es usual que se aplique una metodología de distribución directa de acuerdo a las necesidades de las personas y luego una distribución aleatoria por disponibilidad a la ubicación [34].

\section{Tendencias u oportunidades de investigación}

Esta sección corresponde a la respuesta de la tercera pregunta de investigación sobre tendencias u oportunidades de investigación en los métodos de optimización y mejora de slotting y picking. Una vez analizados los conceptos, aplicaciones y factores que se consideran en el análisis de slotting y picking, se puede concluir que en la mayoría de ellos no consideran técnicas de acomodo dinámico y no especifican las formas cómo se reabastece el almacén, generando así posibles inconvenientes por tiempos de reabastecimiento, capacidad de mano de obra y otras mediciones que podrían alterar el desempeño en forma significativa.

A partir de la revisión de literatura realizada se evidencia la necesidad de desarrollar metodologías de integración de las condiciones mencionadas anteriormente con otros modelos, incluyendo combinación de diseño de almacén con políticas de inventario, teniendo presente la incertidumbre o casos estocásticos, dado que podría considerarse que, aun usando modelos determinísticos, en la práctica pueden llevar a una toma de decisiones que podrían no comportarse acorde a la planeación de la empresa.

Se deberían considerar, además, las dimensiones de los diferentes SKUs para realizar un mejor análisis de ubicación volumétrica y cómo se estaría afectando las variables de desempeño, dado que a pesar de que se cumpla restricciones de ubicación, podría generarse el no uso de espacios o espacios muertos en el sistema de almacenamiento, llevando así a una subutilización y desperdicio de espacio. Idealmente, una vez definidas las restricciones macro, realizar un acomodo con las restricciones máximas de los estantes, y luego adaptar el sistema del almacenamiento de acuerdo con los requerimientos y necesidades que se presenten.

\section{Acomodo dinámico}

En el acomodo dinámico, se requiere desarrollar metodologías que tengan en cuenta las tasas de entrada de producto como criterio para el dimensionamiento de espacios o asignación de los SKUs a los distintos slots disponibles [36-37]. También, considerar las políticas de inventario y el estado actual del mercado, entendiendo que las políticas de inventario pueden variar en el tiempo en relación con las comparaciones de costo de las políticas futuras [35].

\section{Análisis de rutas}

Otra oportunidad de investigación es el análisis de rutas de acuerdo a las capacidades de transporte, cantidad de operarios, condiciones físicas y metodologías de picking [7]. También, analizar cómo se podrían incluir los métodos de selección de rutas de acuerdo con una orden de pedido [38-40], aprovechando las tecnologías de información y comunicación y desarrollando un algoritmo tal que encuentre una ruta válida, poco extensa y que el operario pueda seguir desde algún dispositivo, teniendo así mayor control de los SKUs recolectados [41].

\section{División del almacén por tipo de productos}

En la gestión del almacenamiento se requiere desarrollar metodologías de clasificación que integren criterios de semejanzas, condiciones ambiente ideales, tipo de producto y otras características propias de cada SKU, con el fin de tener presente restricciones más reales al momento de realizar una microlocalización [4, 12]. Estas metodologías 
requieren considerar simultáneamente la clasificación de los SKUs, la asignación de familias a las islas y la microlocalización de cada SKU en las islas [35].

\section{CONCLUSIONES}

El slotting y el picking son actividades fundamentales en el sistema de administración de almacenes y la gestión de cadenas de suministro y pueden representar más de la mitad de los costos de funcionamiento y operación. Por ello, se han desarrollado modelos y metodologías para la asignación adecuada de SKUs en los espacios de almacenamiento y la recogida de los mismos. Sin embargo, la mayoría de los modelos desarrollados se cierran a las condiciones óptimas de aplicación o condiciones sencillas, pasando por alto la variedad de decisiones y diferencias entre almacenes, de acuerdo al tipo de industria, funcionamiento y restricciones.

El slotting y el picking, a pesar de tener fines opuestos, son complementarios y una buena implementación de slotting puede facilitar en gran medida el picking, dado que la ubicación estratégica de los SKUs facilita la recolección de estos, haciéndola eficiente y menos costosa. Por tal motivo, estas dos actividades deben considerarse conjuntamente en la toma de decisiones en la gestión del almacenamiento. El slotting y el picking pueden ser aplicados y adaptados a diversas industrias y operaciones diferentes al almacenamiento, puesto que el manejo adecuado de inventarios permite obtener procesos más eficientes, mayor capacidad de respuesta y un mayor nivel de atención al cliente.

La mayoría de modelos y metodologías analizados en este trabajo, luego de aplicar la metodología de recopilación y clasificación de la evidencia documental encontrada, están basados en condiciones determinísticas y aplicados en almacenes con un numero de referencias relativamente bajo. Se evidencia entonces la necesidad de desarrollar modelos y metodologías de slotting y picking que sean flexibles e incorporen la incertidumbre de posibles variaciones del mercado e, inclusive, cambios repentinos en la industria. En los modelos de administración y localización de SKUs se debe considerar la posibilidad de tener dos o más almacenes ubicados en zonas diferentes y de los cuales depende el desempeño de las actividades de producción.
Aunque el desarrollo de modelos y metodologías de solución de problemas de slotting y picking data de mediados del siglo XX, en la última década se ha desarrollado y aplicado con mayor evolución integrando técnicas heurísticas y de programación con el fin abordar problemas en condiciones más cercanas a la realidad. Por ello, los hallazgos obtenidos en este trabajo constituyen un punto de partida para el desarrollo de futuras investigaciones sobre metodologías y modelos de slotting y picking que integren técnicas avanzadas de modelado analítico en condiciones de alta incertidumbre y en sectores empresariales específicos.

\section{REFERENCIAS}

[1] N. Slack, A. Brandon-Jones and R. Johnston "Operations Management". 8th Ed. Harlow, UK: Pearson. 2016.

[2] S. Chopra and P. Meindl. "Supply Chain Management: Strategy, Planning, and Operation”. 6th Ed. New York: Pearson. 2016.

[3] H. Fourati, H. Idoudi and L.A. Saidane. "Intelligent slots allocation for dynamic differentiation in IEEE 802.15.6 CSMA/ CA". Ad Hoc Networks. Vol. 72, pp. 27-43. 2018. URL: https://doi.org/10.1016/j. adhoc.2018.01.007

[4] N.A. Ribeiro, A. Jacquillat, A.P. Antunes, A.R. Odoni and J.P. Pita. "An optimization approach for airport slot allocation under IATA guidelines". Transp. Res. Part B Methodol. Vol. 112, pp. 132-156. 2018. URL: https://doi.org/10.1016/j.trb.2018.04.005

[5] M.A. Millstein, L. Yang and H. Li. "Optimizing ABC inventory grouping decisions". Int. J. Prod. Econ. Vol. 148, pp. 71-80. 2014. URL: https://doi.org/10.1016/j.ijpe.2013.11.007

[6] A. Kovács. "Optimizing the storage assignment in a warehouse served by milkrun logistics". Int. J. Prod. Econ. Vol. 133, Issue 1, pp. 312-318. 2011. URL: https:// doi.org/10.1016/j.ijpe.2009.10.028

[7] M. Matusiak, R. de Koster and J. Saarinen. "Utilizing individual picker skills to improve order batching in a warehouse". Eur. J. Oper. Res. Vol. 263, Issue 3, pp. 888-899. 2017. URL: https://doi.org/10.1016/j. ejor.2017.05.002

[8] J. Coyle, E. Bardi and J. Langley. "The Management of Business Logistics: A Supply 
Chain Perspective", 7th Ed. Boston: SouthWestern. 2002.

[9] J. Fichtinger, J.M. Ries, E.H. Grosse and P. Baker. "Assessing the environmental impact of integrated inventory and warehouse management". Int. J. Prod. Econ. Vol. 170, pp. 717-729. 2015. URL: https://doi. org/10.1016/j.ijpe.2015.06.025

[10] G. Zhang, T. Nishi, S.D.O. Turner, K. Oga and X. Li. "An integrated strategy for a production planning and warehouse layout problem: Modeling and solution approaches". Omega. Vol. 68, pp. 85-94. 2017. URL: https://doi.org/10.1016/j.omega.2016.06.005

[11] T. Pfeiffer. "A comparison of simple two-part supply chain contracts". Int. J. Prod. Econ. Vol. 180, pp. 114-124. 2016. URL: https:// doi.org/10.1016/j.ijpe.2016.06.023

[12] J. Gu, M. Goetschalckx and L.F. McGinnis. "Research on warehouse operation: A comprehensive review". Eur. J. Oper. Res. Vol. 177, Issue 1, pp. 1-21. 2007. URL: https://doi.org/10.1016/j.ejor.2006.02.025

[13] R. Manzini, Y. Bozer and S. Heragu."Decision models for the design, optimization and management of warehousing and material handling systems". Int. J. Prod. Econ. Vol. 170, pp. 711-716. 2015. URL: https:// doi.org/10.1016/j.ijpe.2015.08.007

[14] Y. Zhang. "Correlated Storage Assignment Strategy to reduce Travel Distance in Order Picking." IFAC-PapersOnLine. Vol. 49, Issue 2, pp. 30-35. 2016. URL: https://doi. org/10.1016/j.ifacol.2016.03.006

[15] C. Ortiz-Astorquiza, I. Contreras and G. Laporte. "Multi-level facility location problems". Eur. J. Oper. Res. Vol. 267, Issue 3, pp. 791-805. 2018.

[16] S. Huang, Q. Wang, R. Batta and R. Nagi. "An integrated model for site selection and space determination of warehouses". Comput. Oper. Res. Vol. 62, pp. 169-176. 2015. URL: https://doi.org/10.1016/j.cor.2014.10.015

[17] B. Kitchenham. "Procedures for Performing Systematic Reviews". Joint Technical Report, Issue TR/SE-0401. Department of Computer Science. Keele University, Australia, p. 28. 2004.

[18] A. Reisman. "How can OR/MS Educators Benefit From Creating and Using Taxonomies?". INFORMS Trans. Educ.
Vol. 4, Issue 3, pp. 55-65. 2004. URL: https:// doi.org/10.1287/ited.4.3.55

[19] F. Bindi, R. Manzini, A. Pareschi and A. Regattieri. "Similarity-based storage allocation rules in an order picking system: An application to the food service industry". Int. J. Logist. Res. Appl. Vol. 12, Issue 4, pp. 233-247. 2009. URL: https://doi. org/10.1080/13675560903075943

[20] K. Derickx. "A comparative study of different storage policies in warehouse management". p. 125.2012.

[21] P.C. Schuur. "The worst-case performance of the Cube per Order Index slotting strategy is infinitely bad - A technical note". Int. J. Prod. Econ. Vol. 170, pp. 801-804. 2015. URL: https://doi.org/10.1016/j.ijpe.2015.05.027

[22] R. Manzini, R. Accorsi, M. Gamberi and S. Penazzi. "Modeling class-based storage assignment over life cycle picking patterns". Int. J. Prod. Econ. Vol. 170, pp. 790-800. 2015. URL: https://doi.org/10.1016/j. ijpe.2015.06.026

[23] Y. Yu, R.B.M. De Koster and X. Guo. "Class-Based Storage with a Finite Number of Items: Using More Classes is not Always Better". Prod. Oper. Manag. Vol. 24, Issue 8, pp. 1235-1247. 2015. URL: https://doi. org/10.1111/poms.12334

[24] X. Guo. "Storage Policies and Maintenance Support Strategies in Warehousing Systems". Springer Theses. Vol. 53, Issue 9, p. 235. 2011.

[25] R.J. Kuo, P.H. Kuo, Y.R. Chen and F.E. Zulvia. "Application of metaheuristics-based clustering algorithm to item assignment in a synchronized zone order picking system". Appl. Soft Comput. J. Vol. 46, pp. 143150. 2016. URL: https://doi.org/10.1016/j. asoc.2016.03.012

[26] B.S. Kim and J.S. Smith. "Slotting methodology using correlated improvement for a zone-based carton picking distribution system”. Comput. Ind. Eng. Vol. 62, Issue 1, pp. 286-295. 2012. URL: https://doi. org/10.1016/j.cie.2011.09.016

[27] H. de Ruijter. "Improved storage in a book warehouse", p. 72, October, 2007. URL: https:// www.semanticscholar.org/paper/Improvedstorage-in-a-book-warehouse-Ruijter-Schuur/ ceb1fdff66c162894698fb960eb159890 $8 \mathrm{~b} 5 \mathrm{e} 372$ 
[28] R.J. Mantel, P.C. Schuur and S.S. Heragu. "Order oriented slotting: a new assignment strategy for warehouses.” Eur. J. Ind. Eng. Vol. 1, Issue 3, p. 301. 2007. URL: https:// doi.org/10.1504/EJIE.2007.014689

[29] R. Accorsi, R. Manzini and F. Maranesi. "A decision-support system for the design and management of warehousing systems". Comput. Ind. Vol. 65, Issue 1, pp. 175186. 2014. URL: https://doi.org/10.1016/j. compind.2013.08.007

[30] P. Wutthisirisart, M.Y. Sir and J.S. Noble. "The two-warehouse material location selection problem". Int. J. Prod. Econ. Vol. 170, pp. 780-789. 2015. URL: https:// doi.org/10.1016/j.ijpe.2015.07.008

[31] P.M. Picard, A. Tampieri and X. Wan. "Airport capacity and inefficiency in slot allocation". Int. J. Ind. Organ. Vol. 62, pp. 330-357. 2019. URL: https://doi.org/10.1016/j. ijindorg.2017.10.003

[32] Y. Fu, L. Song, K.K. Lai and L. Liang. "Slot allocation with minimum quantity commitment in container liner revenue management: A robust optimization approach”. Int. J. Logist. Manag. Vol. 27, Issue 3, pp. 650-667. 2016. URL: ttps://doi. org/10.1108/IJLM-06-2013-0075

[33] M.A. Madas and K.G. Zografos. "Airport slot allocation: From instruments to strategies." J. Air Transp. Manag. Vol. 12, Issue 2, pp. 53-2. 2006. URL: https://doi.org/10.1016/j. jairtraman.2005.08.001

[34] Ministerio De Salud Pública y Asistencia Social-Guatemala. "Modelo de Atención Integral en Salud MSPAS". p. 61. 2010.
[35] B.S.S. Tejesh and S. Neeraja. "Warehouse inventory management system using IoT and open source framework". Alexandria Eng. J. Vol. 57, Issue 4, pp. 3817-3823. 2018. URL: https://doi.org/10.1016/j.aej.2018.02.003

[36] P. Sukhov, M. Batsyn and P. Terentev. "A dynamic programming heuristic for optimizing slot sizes in a warehouse". Procedia Comput. Sci. Vol. 31, pp. 773-777. 2014. URL: https:// doi.org/10.1016/j.procs.2014.05.327

[37] J.A. Pazour and H.J. Carlo. "Warehouse reshuffling: Insights and optimization". Transp. Res. Part E. Vol. 73, pp. 207-226. 2015. URL: http://dx.doi.org/10.1016/j. tre.2014.11.002

[38] U. Pferschy and J. Schauer. "Order Batching and Routing in a Non-Standard Warehouse". Electron. Notes Discret. Math. Vol. 69, pp. 125-132. 2018. URL: https://doi. org/10.1016/j.endm.2018.07.017

[39] F. Yener and H.R. Yazgan. "Optimal warehouse design: Literature review and case study application”. Comput. Ind. Eng. Vol. 129, pp. 1-13. 2019. URL: https://doi. org/10.1016/j.cie.2019.01.006

[40] F. Weidinger. "Picker routing in rectangular mixed shelves warehouses". Comput. Oper. Res. Vol. 95, pp. 139-150. 2018. URL: https:// doi.org/10.1016/j.cor.2018.03.012

[41] C. Theys, O. Bräysy, W. Dullaert and B. Raa. "Using a TSP heuristic for routing order pickers in warehouses". Eur. J. Oper. Res. Vol. 200, Issue 3, pp. 755-763. 2010. URL: https://doi.org/10.1016/j.ejor.2009.01.036

[42] R. Ballou. "Business Logistics: Supply Chain Management”. 5th Ed. Phoenix: Pearson. 2003. 